\title{
Beneficial effects of the fructus Sophorae extract on experimentally induced osteoporosis in New Zealand white rabbits
}

\author{
LIDIJA CHAKULESKA ${ }^{1}$ \\ ALEKSANDAR SHKONDROV ${ }^{2}$ \\ GEORGI POPOV $^{3}$ \\ NADYA ZLATEVA-PANAYOTOVA ${ }^{4}$ \\ RENETA PETROVA \\ MARIYANA ATANASOVA 6 \\ ILINA KRASTEVA2 \\ IRINI DOYTCHINOVA ${ }^{6}$ \\ RUMYANA SIMEONOVA, \\ ${ }^{1}$ Department of Pharmacology, Pharmacotherapy \\ and Toxicology, Faculty of Pharmacy, Medical \\ University of Sofia, 1000 Sofia, Bulgaria \\ ${ }^{2}$ Department of Pharmacognosy, Faculty of \\ Pharmacy, Medical University of Sofia, 1000 \\ Sofia, Bulgaria \\ ${ }^{3}$ Department of Internal Non-infectious Diseases \\ Pathology and Pharmacology, Faculty of \\ Veterinary Medicine, University of Forestry 10 \\ 1756 Sofia, Bulgaria \\ ${ }^{4}$ Department of Surgery and Radiology, Faculty of \\ Veterinary Medicine, University of Forestry, 10 \\ 1756 Sofia, Bulgaria \\ ${ }^{5}$ National Diagnostic and Research Veterinary \\ Institute, Department: National Center of Animal \\ Health, Sofia, Bulgaria \\ ${ }^{6}$ Department of Chemistry, Faculty of Pharmacy \\ Medical University of Sofia, 1000 Sofia, Bulgaria
}

\begin{abstract}
Sophora japonica is a source of several flavonol, flavone and isoflavone glycosides that are reported to positively affect menopausal symptoms including osteoporotic complications. In the present study fructus Sophorae extract (FSE) was administered orally for three months at a dose of $200 \mathrm{mg} \mathrm{kg}^{-1}$ in ovariectomized (OVX) New Zealand rabbits. 3D computed tomography scans and histopathological images revealed microstructural disturbances in the bones of the castrated animals. FSE recovered most of the affected parameters in bones in a manner similar to zoledronic acid (ZA) used as a positive control. The aglycones of the main active compounds of FSE, daidzin, and genistin, were docked into the alpha and beta estrogen receptors and stable complexes were found. The findings of this study provide an insight into the effects of FSE on bone tissue loss and suggest that it could be further developed as a potential candidate for the prevention of postmenopausal osteoporotic complications.
\end{abstract}

Keywords: osteoporosis, Sophora japonica fruits, ovariectomized rabbits, molecular docking, estrogen receptors

Accepted April 7, 2021

Published online April 9, 2021

Osteoporosis (OP) is a common non-communicable disease, affecting more than 200 million individuals worldwide and the leading cause of morbidity and death due to bone fractures (1). Oxidative stress (OS) is considered as the common pathogenic factor for 'menopausal syndrome' that includes several disturbances and diseases, such as postmenopausal osteoporosis. The estrogen decline associated with the menopausal transition is believed to be the 'spark' that triggers OS. A vast body of studies shows that bilateral ovariectomy induces a redox imbalance characterized by increased levels of lipid per-

\footnotetext{
*Correspondence; e-mail address: rsimeonova@pharmfac.mu-sofia.bg
} 
oxidation markers and decreased activity of antioxidant enzymes (2). Some additional factors might contribute to developing osteoporosis. Metabolic syndrome is a constellation of medical conditions consisting of central obesity, hyperglycemia, hypertension, and dyslipidemia, in which each acts on bone tissue in a different way. It may play a key role in the pathogenesis of OP (3).

This investigation is a continuation of our ongoing studies on plant-derived agents that could positively affect osteoporosis, in which we described the bone-protective effects of Ruscus aculeatus extract in ovariectomized rats (4). To carry out the present experiment, an extract from fruits of Sophora japonica, which is known to contain isoflavones, which have been shown to provide estrogen-like effects in menopause, was used. Sophora japonica L. (Fabacaeae) is a source of natural isoflavones that could help alleviate postmenopausal symptoms. In a randomized double-blind placebo-controlled clinical trial (5) it was shown that the consumption of the fructus Sophorae extract (FSE) possessed beneficial effects on the postmenopausal symptoms in women and may also decrease the risk of fracture in osteoporotic patients. Isoflavonoids have been the focus of studies on natural phytoestrogens and the complex of isoflavone glycosides extracted from fructus Sophorae was listed as a potential cause for the beneficial effects (6). These bioactive substances have been shown to exert potential antiosteoporotic effects in both in vitro and in vivo models in rats (7-9), but to our knowledge, they were not investigated in animals with bigger bones. In this case, however, we focused on another type of experimental animal - rabbits. They have some advantages over more commonly used rats, including bigger weight and thus, larger bones, similar to those of big mammals.

For experimental investigation of postmenopausal osteoporosis, in addition to the common ovariectomy (OVX)-induced rat model of estrogen deficiency, the American Food and Drug Administration (FDA) recommends the use of larger species including rabbits, sheep, goats, dogs, and swine, which have the ability to remodel their bone cortices $(10,11)$. Rabbits are the smallest commonly employed laboratory animals with well-defined spontaneous cortical remodelling similar to humans, shorter remodelling period than larger animal models, and relatively rapid skeletal maturation (by six to nine months) (11).

In addition, we decided to use another imaging method for the detection of bone abnormalities, a computed tomography (CT) scanner, which generates high-quality and more accurate images of the bone areas we are interested in; CT scanner focuses on the target area better than the X-ray.

Thus, the aim of the present study was to investigate the effects of FSE on the skeletal system of rabbits with OVX-induced osteoporosis by different imaging techniques and to compare the effects of the extract with the well-known anti-osteoporotic drug, zoledronic acid (ZA).

\section{EXPERIMENTAL}

\section{Solvents and reagents}

Dichloromethane and methanol for extraction were purchased from Sigma-Aldrich, (Germany) and were of analytical grade. Acetonitrile (Sigma-Aldrich), o-phosphoric 
acid and Milli-Q ${ }^{\circledR}$ water (Merck, Germany) were of HPLC grade. Daidzin CRS and genistin CRS (purity over $95 \%$ ) were purchased from Chromadex (USA). Ketamine hydrochloride, xylazine, and zoledronic acid were purchased from a pharmacy in Sofia, Bulgaria. Commercially available kits (see below) were used for measurement of calcium, phosphorus, osteocalcin, Beta-CrossLaps, and parathormone.

\section{Plant material}

Fruits of Sophora japonica (Fabaceae) were collected from the town of Bitola, R. N. Macedonia in September 2018. The species was identified by Ilina Krasteva and a voucher specimen was deposited in the herbarium of the Faculty of Pharmacy, Medical University of Sofia, Bulgaria (FF-127-19).

\section{Extraction}

The air-dried and pulverized fruits $(500 \mathrm{~g})$ were defatted with dichloromethane $(5 \times 2 \mathrm{~L})$ to remove the lipophilic compounds and then exhaustively extracted with $80 \%$ $\mathrm{MeOH}$ by percolation. The resulting methanol extract was evaporated to eliminate the solvent, lyophilized and marked as FSE (190 g).

\section{HPLC analysis}

The isoflavone content of FSE was analysed by a known HPLC method (12). The method was re-validated. Daidzin CRS and genistin CRS (Fig. 1) were dissolved in methanol $\left(1 \mathrm{mg} \mathrm{mL}^{-1}\right)$ and serial dilutions were made to make the calibration curves. A Young Lin 9100 HPLC system (Hogyedong, Korea) equipped with YL 9101 vacuum degasser, YL 9110 quaternary pump, YL 9131 column compartment, YL 9160 PDA detector, 7725i manual injector and software Clarity (v. 2) was used. Separations were performed on a pre-packed ODS column Luna ${ }^{\circledR}(100 \AA, 250 \times 4.6 \mathrm{~mm}, 5 \mu \mathrm{m}$, Phenomenex, USA), coupled with Security Guard ${ }^{\circledR}$ octadecylsilyl cartridge (Phenomenex). The binary solvent system consisted of $o$-phosphoric acid $(0.1 \%)$ and acetonitrile. The flow rate was $1.0 \mathrm{~mL} \mathrm{~min}^{-1}$. The temperature was set at $40^{\circ} \mathrm{C}$. The injected volume was $20 \mu \mathrm{L}$. All chromatograms were recorded at $260 \mathrm{~nm}$. All injections were made in triplicates and the mean value was calculated.

\section{Animals}

The studies were performed on sixteen skeletally mature, female New Zealand white rabbits, six-month-old, weighing $2.50 \pm 0.34 \mathrm{~kg}$. The experimental animals were<smiles>O=c1c(-c2ccc(O)cc2)coc2cc(O[C@@H]3O[C@H](CO)[C@@H](O)[C@H](O)[C@H]3O)cc(O)c12</smiles>

Daidzin

Genistin

Fig. 1. Structures of daidzin and genistin. 
purchased from the National Breeding Center in Slivnitsa, Bulgaria, and were kept under standard conditions in metal cages, at a temperature of $20-25^{\circ} \mathrm{C}$, mode $12 \mathrm{~h} / 12 \mathrm{~h}$ light/dark. The standard commercial pelleted feed and drinking water were available ad libitum during the entire experimental period of 90 days. Seven-day acclimatization of the animals was allowed before the beginning of the experiment, with regular monitoring of their health condition by a veterinarian. The rabbits were housed, maintained and euthanized in accordance with the relevant international rules and recommendations as stated in the European Convention for the Protection of Vertebrate Animals used for Experimental and other Scientific Purposes (ETS 123). The Animal Care Ethics Committee approved the study protocol and Ethics clearance (No 233 from 09/02/2019) was issued for the study by the Bulgarian Agency for Food Safety.

\section{Study design}

Sixteen female New Zealand rabbits were divided into individual cages at random into four groups. After the acclimatization period, on the $7^{\text {th }}$ day, blood from the ear vein of all rabbits was collected to establish the base level of all investigated biochemical parameters. Thereafter, an abdominal resection without removing the ovaries was performed in four rabbits. They served as a control group. The other 12 animals underwent bilateral ovariectomy (OVX) (13).

One week after the surgical procedure, the animals were divided into groups $(n=4$ each) as follows: group 1 - control group of rabbits (sham) with free access to drinking water, group 2 - OVX control rabbits with free access to drinking water, group 3 - OVX rabbits treated with FSE $\left(200 \mathrm{mg} \mathrm{kg}^{-1} \mathrm{bm}\right)$ dissolved in drinking water and administered per os for a period of 90 days, group 4 - OVX rabbits treated once subcutaneously with zoledronic acid (ZA) as a positive control $\left(100 \mu \mathrm{g} \mathrm{kg}^{-1} \mathrm{bm}\right)$ diluted with saline (14).

On the $7^{\text {th }}$ and $45^{\text {th }}$ day, after fasting, blood from the ear vein from all animals was taken to examine the biochemical markers of calcium homeostasis and bone turnover. From $85^{\text {th }}$ to $90^{\text {th }}$ day, blood was taken again for biochemical analysis. Thereafter, the animals were anaesthetized via intramuscular injection with the combination of 90

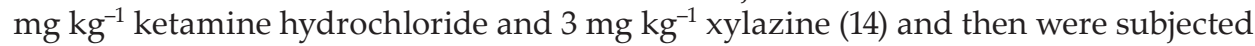
to computed tomography of the vertebral, pelvic and long bones areas. On day 91, all animals were euthanized by $\mathrm{CO}$ respiration. The vertebral, pelvic and long bones were excised and prepared for histological analysis.

\section{Markers of calcium homeostasis}

On days 7, 45, and 85 blood from the ear vein was collected in tubes containing a clot activator. After centrifugation at 3,000 $\times g$ for $10 \mathrm{~min}$, the serum was separated and stored at $-20^{\circ} \mathrm{C}$ for evaluation of investigated parameters.

Calcium and phosphorus were measured using commercially available kits for biochemical analyzer Mindray - BP 120 (China). Parathyroid hormone (PTH) levels were analyzed using electrochemiluminescence immunoassay "ECLIA" with kits for immunoassay analyzer (Cobas-Roche, Switzerland). 


\section{Markers of bone turnover}

Osteocalcin (OC), a marker of bone formation, and Beta-CrossLaps ( $\beta$-CTx), a marker of bone resorption, were analyzed on the $7^{\text {th }}, 45^{\text {th }}$ and $85^{\text {th }}$ days using "ECLIA" with kits for immunoassay analyzer (Cobas-Roche).

\section{Computed tomography}

Evaluation of bone density was performed under general anaesthesia with a CT Picker ${ }^{\circledR}$ CT PQ 5000 scanner. The CT scan machine was manipulated under $60 \mathrm{kV}$ and $180 \mu \mathrm{A}$. The scanned images of each specimen were analyzed on the computer using the DICOM Viewer ${ }^{\circledR}$ software.

Bone density measurements were made at three points in different segments of the caudal skeleton as follows: pelvic area (Pel) - in the dorsal middle area (left and right), in the area of the os ileum (left and right); the proximal segment of the femur $(\mathrm{PF})$ - in the trochanter area, femoral neck, and proximal compact zone; vertebrae - in the corpus vertebrae area and laterodorsal parts of arcus vertebrae (left and right) of the fourth $\left(\operatorname{Ver}_{4}\right)$, fifth $\left(\operatorname{Ver}_{5}\right)$, sixth $\left(\operatorname{Ver}_{6}\right)$ and seventh $\left(\operatorname{Ver}_{7}\right)$ vertebrae. The mean bone density values in Hounsfield units (HU) of the bone were recorded using a CT Picker ${ }^{\circledR}$ CT PQ 5000 scanner. The results obtained are presented as mean values measured in HU (Hounsfield scale).

\section{Histological examination of rabbits' bone}

Femurs and lumbar vertebrae of the rabbits from all groups were removed post mortem and fixed in $10 \%$ buffered formalin for $48 \mathrm{~h}$. Fixed bones were placed in a decalcification solution (formic acid) for 14 days and then processed according to the classical paraffin method. The sections were stained with hematoxylin and eosin (H\&E). Histological changes were observed and photo-documented with a light microscope with a built-in camera Euromex BioBlue (Belgium).

\section{Molecular docking of genistein and daidzein}

The aglycones daidzein and genistein were studied because gastrointestinal hydrolysis of daidzin and genistin occurs after p.o. administration (4). The 3D structures of daidzein (DAI) and genistein (GEN) were retrieved from PubChem and docked into the ligand-binding site of the alpha and beta forms of the human estrogen receptor (ER $\alpha$ and ER $\beta$ ) derived from RCSB (pdb ids: $1 \times 7 \mathrm{r}, R=2.0 \AA$ and $1 \times 7 \mathrm{j}, R=2.3 \AA$, resp.) (15). Docking was performed by GOLD v. 5.2.2 (CCDC Ltd., Cambridge, UK) at the following settings: evaluation function ChemPLP, flexible ligand and binding site, radius $6 \mathrm{~A}$ at the binding site, included a water molecule (HOH7 for ER $\alpha$ and $\mathrm{HOH} 1$ for ER $\beta$ ) and 100 GA cycle. The residues from the binding site that are in close proximity to the bound ligand were set to be flexible. For Er $\alpha$, these were Met343, Leu346, Leu349, Glu353, Met388, Leu391, Arg394, Phe404, Ile424 and His524; for $\operatorname{Er} \beta$, these were Met295, Leu298, Leu301, Glu305, Met340, Leu343, Arg346, Phe356, Ile376 and His475. Genistein from the X-ray structures was used as a reference molecule in both ERs. 


\section{Statistical analysis}

Statistical analysis was performed using 'MEDCALC', v. 12.3 (MedCalc Software, Belgium). The significance of the experimental data was assessed using the non-parametric Mann-Whitney $U$ test. Values of $p \leq 0.05$ were considered statistically significant.

\section{RESULTS AND DISCUSSION}

\section{Phytochemical analysis of FSE}

HPLC analysis of FSE was performed by modified method of Krenn and Pötsch (12). Calibration curves were constructed for each reference substance and the equations and coefficients of determination were obtained: $R^{2}=0.9996$ for genistin $\left(t_{\mathrm{R}}=\right.$ $18.853 \pm 0.236 \mathrm{~min})$ and $R^{2}=0.9998$ for daidzin $\left(t_{\mathrm{R}}=16.047 \pm 0.343 \mathrm{~min}\right)$. Limits of detection were: $0.0001 \mathrm{mg} \mathrm{mL}^{-1}$ (genistin) and $0.0002 \mathrm{mg} \mathrm{mL}^{-1}$ (daidzin).

Peaks corresponding to daidzin and genistin were identified in the chromatogram of FSE (Fig. 2). Mean retention times, as well as UV spectra of the compounds (recorded by the PDA detector) in the sample, were consistent with those of the reference substances. It was found that $1 \mathrm{mg}$ of FSE contained $0.057 \mathrm{mg}$ daidzin and $0.085 \mathrm{mg}$ genistin. The content of the isoflavonoid glycosides, calculated as per $1 \mathrm{~g}$ dry plant material was $0.03 \mathrm{~g}$ daidzin and $0.05 \mathrm{~g}$ genistin.

\section{Molecular docking of genistein (GEN) and daidzein (DAI) into the estrogen receptors}

The best-scored docking poses of GEN and DAI into ER $\alpha$ and ER $\beta$ are presented in Fig. 3. According to the ChemPLP scores, both molecules bind better to the ER $\beta$

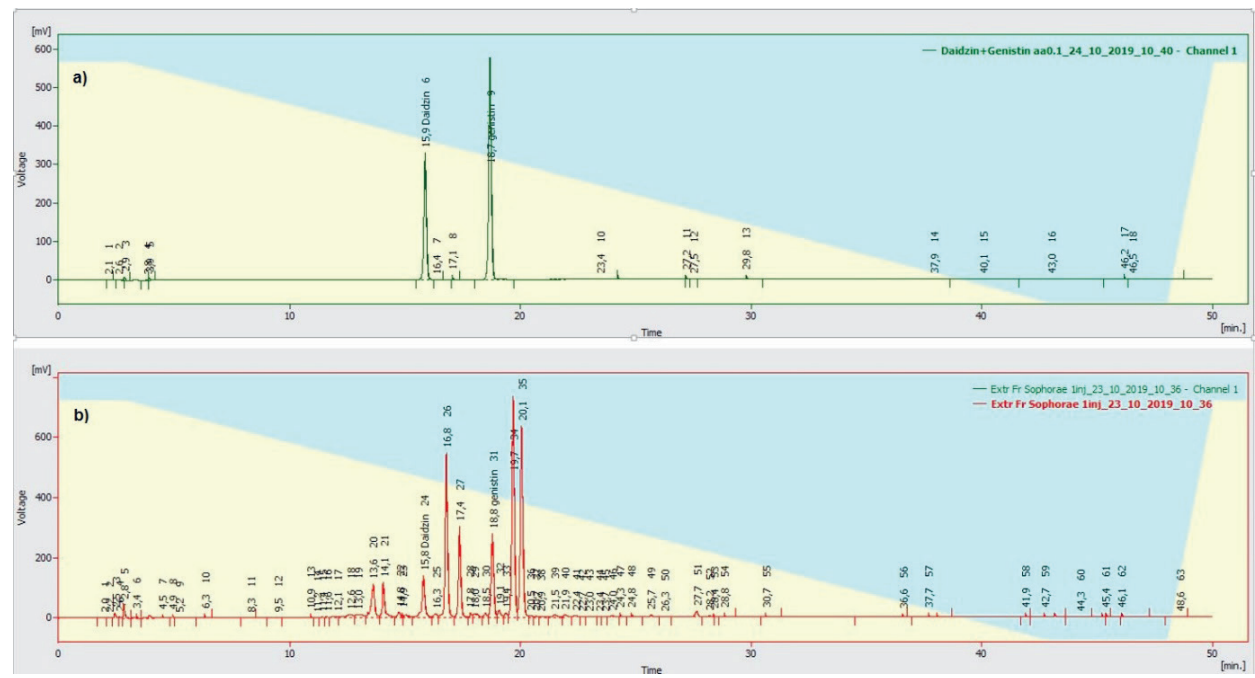

Fig. 2. HPLC chromatograms of: a) standard substances daidzin and genistin (a mixture, $0.1 \mathrm{mg} \mathrm{mL}^{-1}$ of each) and b) of FSE, with peaks corresponding to daidzin (15.8 min) and genistin (18.8 min). Other peaks were not identified. 
a)

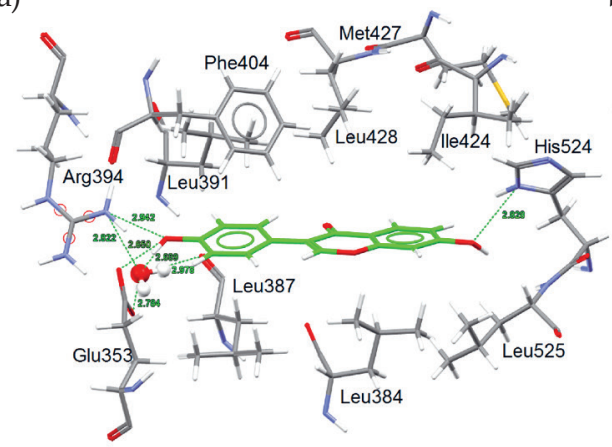

c)

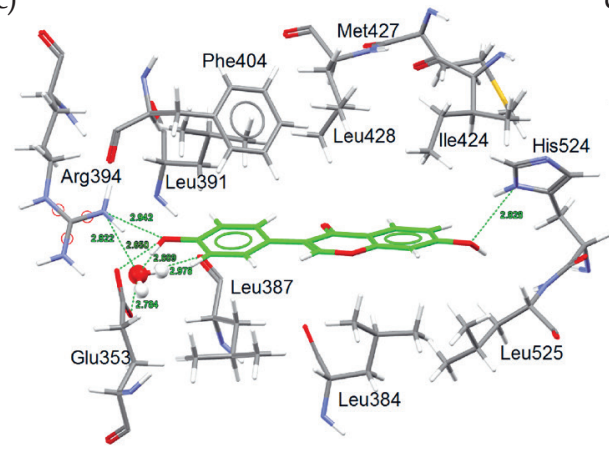

b)

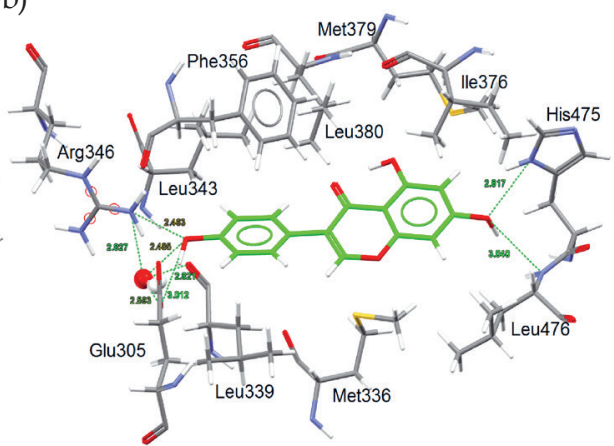

d)

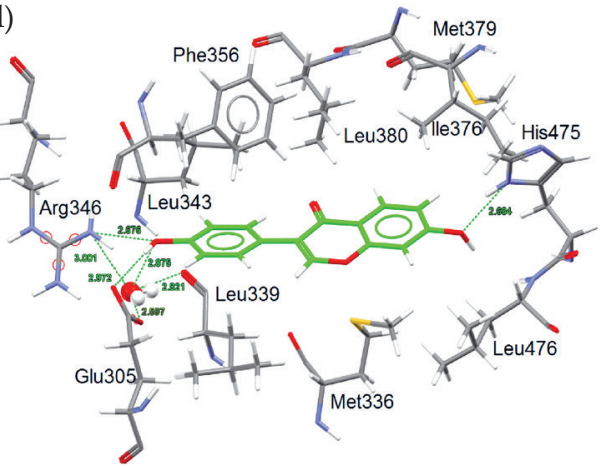

Fig. 3. The best-scored docking poses and interactions of GEN and DAI with ER $\alpha$ (a and c) and ER $\beta$ (b and d), resp. Hydrogen bonds are shown as green dots. The structural water molecule is presented in a "ball and stick" style.

receptor than to the $\operatorname{ER} \alpha$ (Table I). The ER binding site is an oval hydrophobic pocket with polar residues at both ends. The two molecules bind in a similar way to both receptors. The isoflavone moiety lies tangentially on the hydrophobic pocket, whereas the $\mathrm{OH}$ groups at both ends form hydrogen bonds with the polar residues. The phenolic

Table I. ChemPLP fitness scores, piecewise linear potential scores S(PLP) and root mean square deviations (RMSD) for genistein (GEN) and daidzein (DAI) docked into estrogen receptors ${ }^{a}$

\begin{tabular}{ccccccc}
\hline \multicolumn{3}{c}{ ER $\alpha$} & \multicolumn{3}{c}{ ER $\beta$} \\
\hline Compound & ChemPLP & RMSD $(\AA)$ & S(PLP) & ChemPLP & RMSD $(\AA)$ & S(PLP) \\
\hline GEN & 87.70 & 1.342 & -75.74 & 93.49 & 1.299 & -85.26 \\
DAI & 88.28 & 1.233 & -76.35 & 93.63 & 1.299 & -82.26 \\
\hline
\end{tabular}

a The bound GEN from the X-ray structures was used as a reference ligand. ER $\alpha$ - estrogen receptor alpha form; ER $\beta$ - estrogen receptor beta form; ChemPLP - GOLD ChemPLP fitness score (ChemPLP is the name of the function; GOLD is the protein-ligand docking software), RMSD - a root mean square deviation of the docked pose according to the crystallographic structure, S(PLP) - a piecewise linear potential score included in ChemPLP fitness score 
$\mathrm{OH}\left(4^{\prime}-\mathrm{OH}\right)$ forms three hydrogen bonds with Glu353 and Arg394 from ER $\alpha$ and with Glu305 and Arg346 from ER $\beta$. Additionally, it binds to the structural water molecule that forms a dense network of hydrogen bonds between the ligand and ER. The isoflavone 7-OH group forms a hydrogen bond with His524 from ER $\alpha$ and with His475 from ER $\beta$. The same group in GEN makes an additional hydrogen bond with Leu476 on ER $\beta$. The isoflavone 5-OH group of GEN does not form any hydrogen bonds.

The higher ChemPLP scores for ER $\beta$ are coming from the higher S(PLP) values. The term S(PLP) in ChemPLP evaluates the steric complementarity between ligand and receptor (16). More negative values correspond to better complementarity. The results are in good agreement with the 40 times higher selectivity of GEN to ER $\beta$ (15). The estrogenic potency of phytoestrogens is associated mainly with ER $\beta$ activation triggering many of the biological responses, including osteogenic effects (17). On the other side, most of the side-effects of estrogens are associated with the activation of ER $\alpha$ (18). The better binding to ER $\beta$ could be a beneficial property of GEN and DAI.

\section{Body mass changes}

Throughout the experiment, all animals gained weight. OVX rabbits gained $47 \%$ $(p<0.05)$ compared with the initial value at the beginning of the experiment. At the end of the experimental period, the highest body mass gain (BMG) was observed in OVX rabbits by $29 \%(p<0.05)$, compared to the BMG of the control group. When compared to the OVX group at the end of the experiment, the groups treated with FSE and ZA showed significantly lower BWG of 24 and $22 \%$, resp.

The decreased level of estrogens induced by OVX is linked to body mass increase because estrogens and estrogen receptors regulate various aspects of glucose and lipid metabolism (19). The results of this study are in line with the meta-analysis, performed by Akhlaghi and co-workers (20) showing that isoflavones may reduce body mass index (BMI) in women.

\section{Parameters of calcium homeostasis and bone turnover markers}

The changes in parameters of calcium homeostasis and bone turnover markers are shown in Table II. On the $7^{\text {th }}$ day, all measured parameters were similar to the control levels. No differences were observed between the groups. On the $45^{\text {th }}$ day, the levels of calcium were higher $(p<0.05)$ in untreated and all treated OVX rabbits than in the sham control group. In the OVX, FSE and ZA groups calcium level was higher by 35,26 and $20 \%$, resp., compared to the controls $(p<0.05)$. Calcium concentrations in FSE and ZA-treated groups were not statistically different from the OVX group on the $45^{\text {th }}$ day. At the end of the experiment, calcium concentrations in OVX, FSE and ZA-treated groups were still significantly higher $-42,33$ and $31 \%$ resp., compared with the sham-controls and similar to the OVX group. The increased bone turnover following estrogen deprivation after OVX is one of the reasons for higher calcium levels. Probably the observed hypercalcemia suppressed the secretion of the parathyroid hormone (PTH) which was not detectable in this study (Table II).

Phosphorus level, measured as phosphates, was higher $(p<0.05)$ by 58 and $50 \%$ in OVX and FSE-treated group, resp., compared to the control group. The bone turnover rate was probably higher than in the control and ZA group, leading to an increased 


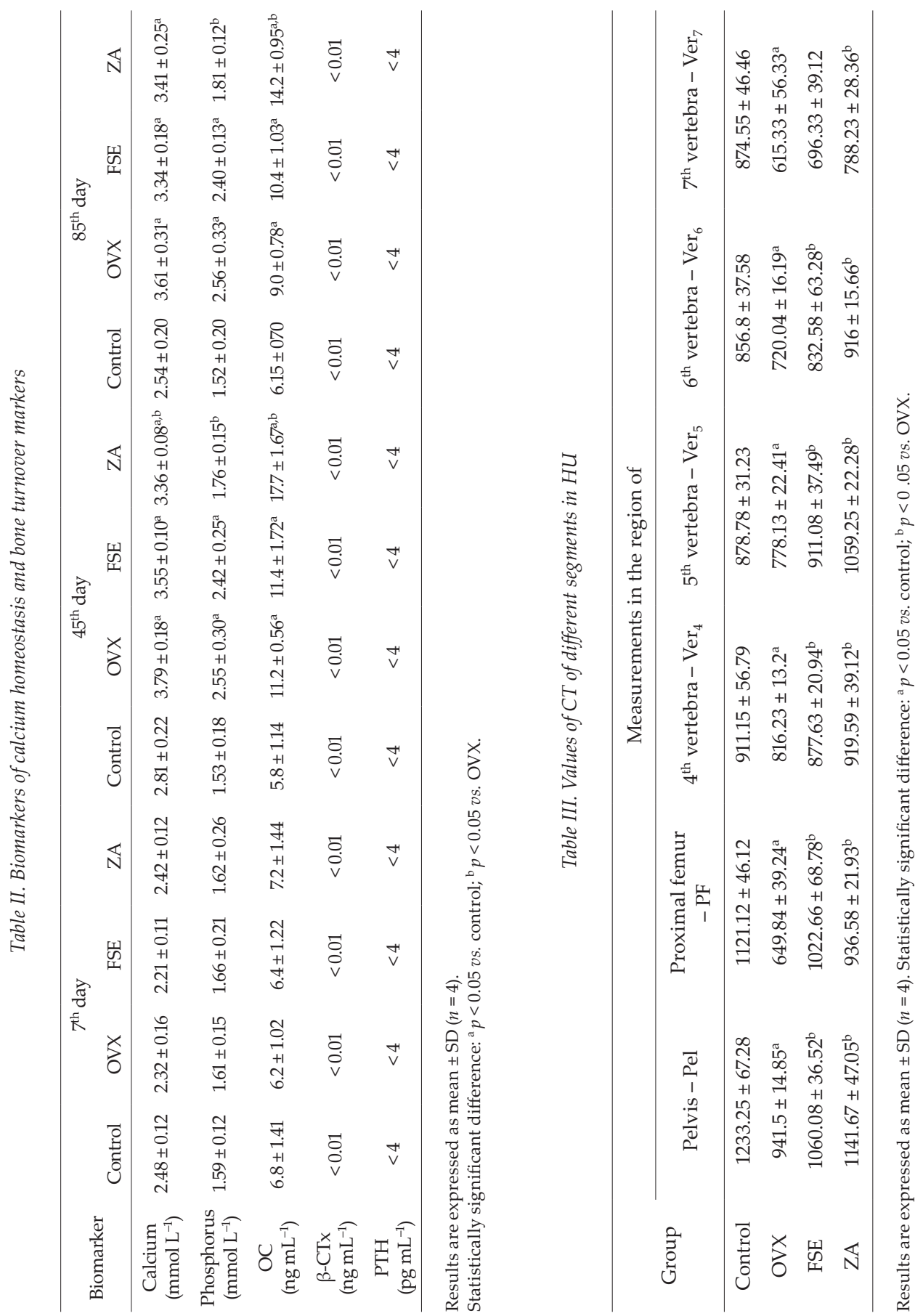


blood phosphorus level in both groups. Treatment of OVX rats with ZA $\left(100 \mu \mathrm{g} \mathrm{kg}{ }^{-1}\right)$ decreased significantly the phosphorus level by $31 \%$, compared to the OVX group and restored this marker to near the control level, which could mean that the drug more effectively incorporates phosphorus into bones. At the end of the experimental period, the results for phosphorus levels were similar to those of the $45^{\text {th }}$ day. OVX and FSE-treated groups had significantly higher phosphorus level with 68 and $37 \%$, resp., than the control. On the $85^{\text {th }}$ day of the experiment, ZA decreased significantly the phosphorus level by $36 \%$, compared to the OVX group (Table II). This decrease was not seen in the FSE-treated group.

On the $45^{\text {th }}$ day the osteocalcin levels were higher by 93,97 and $205 \%$ in OVX, FSE, and ZA group, resp., compared to the sham control rabbits $(p<0.05)$. This trend persisted until day 85, when this marker was 46,68 and $130 \%$, higher in the OVX, FSE and ZA groups resp., compared to the control group. These results are in accordance with the fact that osteocalcin levels are increased in metabolic bone diseases with increased bone or osteoid formation, including osteoporosis (21). It is the most abundant non-collagen protein in the bone matrix, where it regulates mineralization and is produced by mature osteoblasts during bone formation (22). In ZA treated group, as opposed to the FSE group, osteocalcin level was higher for $58 \%(p<0.05)$ than in the OVX only group on the $45^{\text {th }}$ as well as the $85^{\text {th }}$ day. This probably means that the ZA stimulates bone re-mineralization in osteoporotic animals more efficiently than FSE.

The levels of the parathyroid hormone (PTH) and Beta-CrossLaps ( $\beta$-CTx) were below the detection limit of the method used in all experimental groups (Table II). It might be speculated that this is due to the hypercalcemia from one side, and the predominance of the process of bone formation than the process of resorption from another side. Further investigations are needed in order to explain why this happened also in the control animals.

\section{Computed tomography}

The differences in the bone structure of the three main regions of interest [pelvic area (Pel), the proximal segment of the femur $(\mathrm{PF})$ and vertebrae (Ver)] are presented in Table III.

Data show that the highest $(p<0.05)$ bone density in all studied segments was found in the group treated with ZA (Pel-1141.67 HU; PF - $936.58 \mathrm{HU} ; \mathrm{Ver}_{4,5,6,7}-1069.32$ $\mathrm{HU}$ ). These findings are consistent with the results obtained by Öztürk et al. (14) who found that ZA stimulated bone formation and decreased the relapse ratio after expansion in the sagittal suture of rats. Nevertheless, FSE also presented similar well-defined protective bone microstructural effects that were visible on the histomorphological photomicrographs and CT scanner (Figs. S1-S3). The most prominent effect of FSE on bone mass density (BMD) was seen in the proximal region of the femur (Fig. S2), where BMD was $9 \%$ higher than in the ZA group. Generally, the most pronounced effects on BMD were seen on the proximal region of the femur (Table III, Fig. S2). OVX decreased BMD significantly by $42 \%$, compared to the control group, whereas FSE and ZA increased BMD by 57 and $44 \%$, resp., compared to the OVX group. Most of the fractures in postmenopausal women appear in the region of the hip $(1,4)$, so FSE could be a good candidate for the prevention of fractures in this bone region. 
The weakest effect on bone density was seen in the region of Ver4. BMD decreased by $10 \%$ only in castrated animals, compared with the controls. FSE and ZA increased BMD in the $4^{\text {th }}$ vertebrae by 8 and $13 \%$, compared to OVX animals (Table III, Fig. S3).

From the computed tomography examinations, it was found that FSE showed boneprotective effects, although less pronounced than ZA. Compared to non-treated castrated rabbits, FSE treatment significantly increased bone density as follows: in the pelvic region - by $13 \%$, in the proximal femur by $57 \%$, in the $4^{\text {th }}$ vertebra - by $8 \%$, in the $5^{\text {th }}$ vertebra - by $17 \%$, in the $6^{\text {th }}$ vertebra by $16 \%$, and in the $7^{\text {th }}$ vertebra by $13 \%$ (Table III).

\section{Histological examination of the bones of rabbits}

Osteoporosis rabbit' models hold great potential as platforms for advancing our understanding of cortical remodelling and associated bone porosity (9). Microscopic examination of the lumbar vertebrae and femurs from the control animals revealed
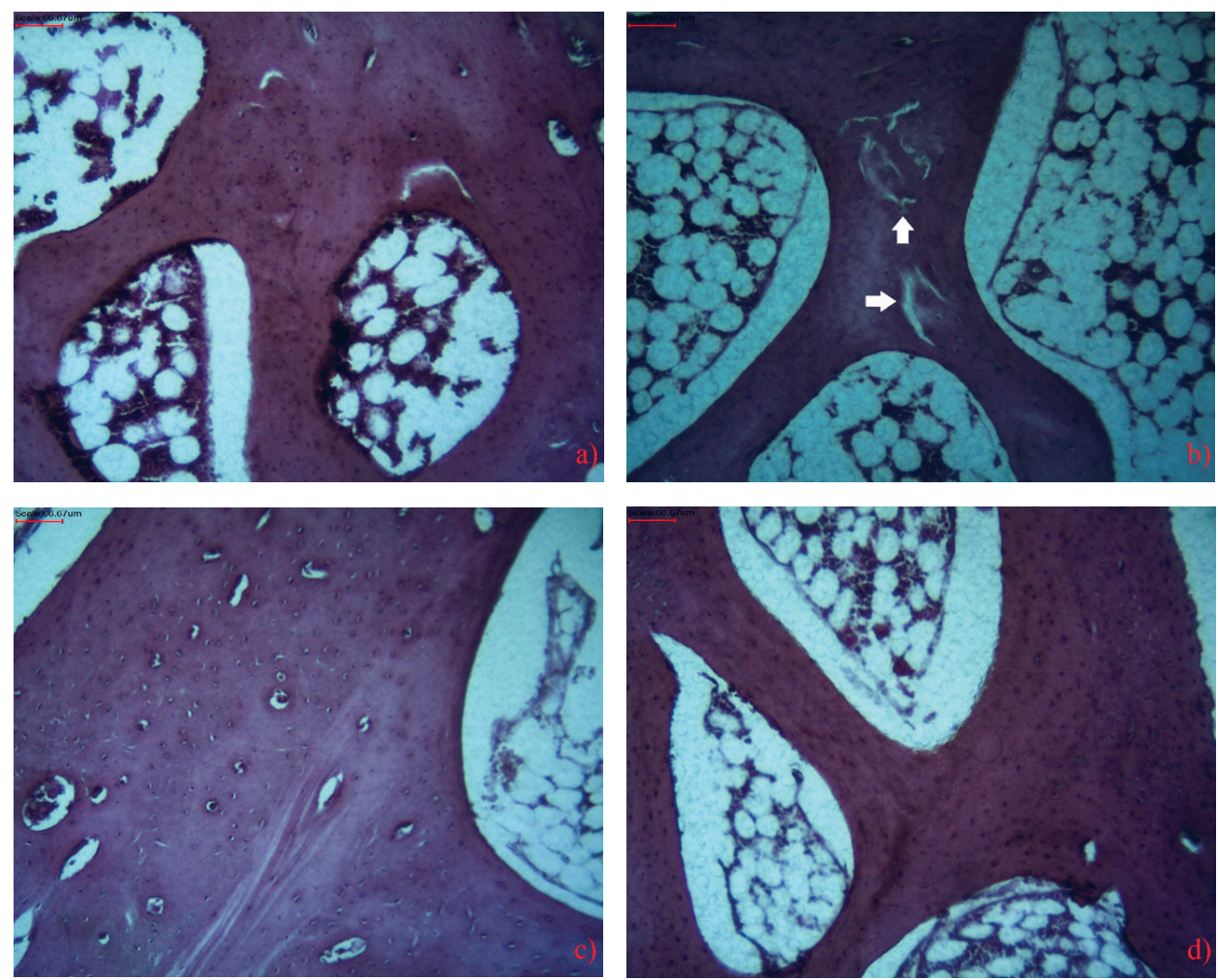

Fig. 4. Photomicrographs of the histological specimens of the lumbar vertebrae of rabbits (H\&E staining). a) Control group: normal histoarchitectonics of bone structure (100×); b) rabbit with ovariectomy: thinning of the lamellar bone tissue with the presence of destructive foci (arrows) (100×); c) rabbit treated with FSE: normal histological structure of the bone with signs of the regenerative process $(100 \times)$; d) rabbit treated with ZA: unaltered histological structure of the bone $(100 \times)$. 
normal histological parameters, expressed in unaltered spongious substance and compact tissue, as well as normal shape, size, and density (Figs. 4a and 5a). In castrated rabbits, thinning of the lamellar structure was found in vertebral bodies and femoral diaphysis. In some areas of the vertebrae, destructive changes were detected, expressed in the disintegration of the bone tissue (Fig. 4b). Similar changes with evidence of osteodystrophy were observed in the femurs (Fig. 5b). In animals treated with FSE and ZA, a preserved microscopic bone structure was observed. In some areas, recovery processes were found, expressed in the ossification of the vertebrae bodies and normal histological structure of the lamellar component (Figs. 4c, d). The same histological features were observed in the femoral diaphysis. Significant regenerative processes were found in some areas, expressed in mineralization and ossification of the femurs with properly formed lamellar structure (Figs. 5c, d).

Isoflavonoids, such as daidzein and genistein, showed therapeutic effects in the case of osteoporosis. Yoon and collaborators (23) showed that genistein is the most
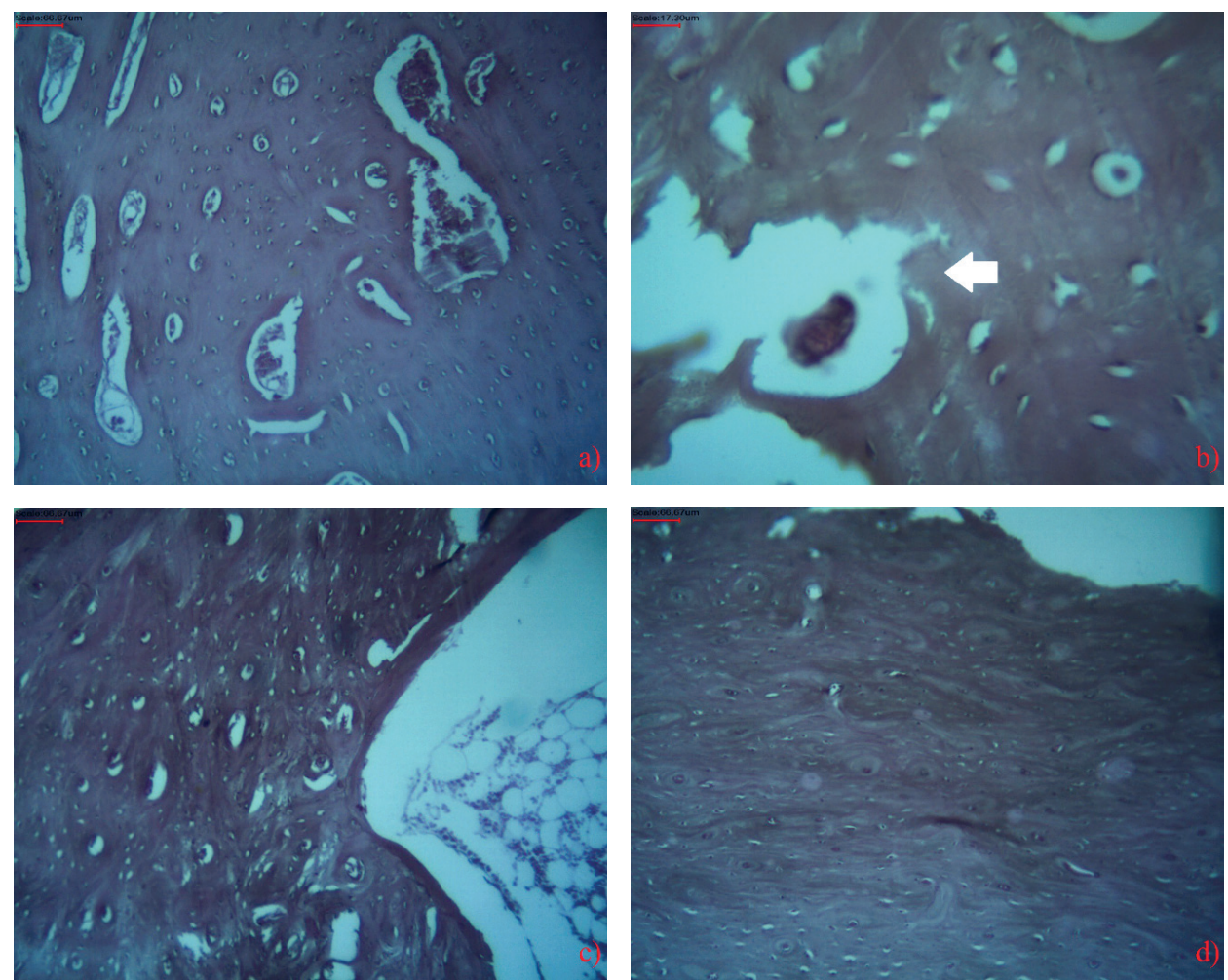

Fig. 5. Photomicrographs of the histological specimens of the right femurs of the rabbits (H\&E staining). a) Control group: normal histological structure of the bone (100×); b) rabbit with ovariectomy: lytic and destructive changes in the bone structure (arrow) (400×); c) rabbit from FSE group: normal histological structure of the bone with signs of mineralization and ossification (100×); d) rabbit from ZA group: normal histological architecture of bone with signs of regeneration (100×). 
abundant compound found in extracts from fruits of $S$. japonica and a key phytoestrogen with osteogenic activity. Moreover, this team studied the effects of genistein in vitro on cell lines, which supports our discussion and allows us to extrapolate their results to the present in vivo findings, especially with larger laboratory animals such as rabbits, which have advantages over rats, as we have already clarified above. Some other experimental studies concluded that daidzein is even more efficient than genistein in preventing ovariectomy-induced bone loss in rats (24). Nevertheless, there is limited information on the activity of extracts, containing the glycosidic forms of these isoflavonoids. That is why we investigated the FSE standardized by its glycosidic content.

\section{CONCLUSIONS}

In conclusion, our findings suggest that FSE demonstrates a significant, but less pronounced, bone protective effect in OVX-induced osteoporotic rabbits compared to zoledronic acid. FSE stimulates osteoblastogenesis and bone formation by maintaining calcium-phosphorus homeostasis and by increasing bone mineral density. In silico predicted ER binding affinity of the constituents of FSE, genistin and daidzin, could also be a reason for the ameliorative, estrogen-like effects of the extract from fruits of S. japonica.

Supplementary material available upon request.

\section{REFERENCES}

1. L. Chakuleska, R. Simeonova and N. Danchev, Pharmacotherapy costs and medicines reimbursement policies of osteoporosis in the Republic of Bulgaria and Republic of North Macedonia, Pharmacia 67 (2020) 199-207; https://doi.org/10.3897/pharmacia.67.e55835

2. G. Bonaccorsi, I. Piva, P. Greco and C. Cervellati, Oxidative stress as a possible pathogenic cofactor of post-menopausal osteoporosis: Existing evidence in support of the axis oestrogen deficiency-redox imbalance-bone loss, Indian J. Med. Res. 147 (2018) 341-351; https://doi:10.4103/ijmr. IJMR_524_18

3. S. K. Wong, K.-Y. Chin, F. H. Suhaimi, F. Ahmad and S. Ima-Nirwana, The relationship between metabolic syndrome and osteoporosis: A review, Nutrients 8 (2016) Article ID 347; https://doi. org/10.3390/nu8060347

4. L. Chakuleska, R. Michailova, A. Shkondrov, V. Manov, N. Zlateva-Panayotova, G. Marinov, R. Petrova, M. Atanasova, I. Krasteva, N. Danchev, I. Doytchinova and R. Simeonova, Bone protective effects of purified extract from Ruscus aculeatus on ovariectomy-induced osteoporosis in rats, Food Chem. Toxicol. 132 (2019) Article ID 110668; https://doi.org/10.1016/j.fct.2019.110668

5. J. Lee, K. W. Kim, H. K. Kim, S. W. Chae, J. C. Jung, S. H. Kwon and C. H. Rheu, The effect of rexflavone (Sophorae fructus extract) on menopausal symptoms in postmenopausal women: a randomized double-blind placebo controlled clinical trial, Arch. Pharm. Res. 33 (2010) 523-530; https:// doi.org/10.1007/s12272-010-0405-0

6. X. He, Y. Bai, Z. Zhao, X. Wang, J. Fang, L. Huang, M. Zeng, Q. Zhang, Y. Zhang and X. Zheng, Local and traditional uses, phytochemistry, and pharmacology of Sophora japonica L. A review, J. Ethnopharmacol. 187 (2016) 160-182; https://doi.org/10.1016/j.jep.2016.04.014

7. S. S. Joo, H. C. Kang, M. W. Lee, Y. W. Choi and D. I. Lee, Inhibition of IL-1beta and IL-6 in osteoblast-like cell by isoflavones extracted from Sophorae fructus, Arch. Pharm. Res. 26 (2003) 10291035; https://doi.org/10.1007/bf02994754 
L. Chakuleska et al.: Beneficial effects of the fructus Sophorae extract on experimentally induced osteoporosis in New Zealand white rabbits, Acta Pharm. 72 (2022) 289-302.

8. S. S. Joo, S. H. Kwon, K. W. Hwang and D. I. Lee, Improvement of menopausal signs by isoflavones derived from Sophorae fructus in ovariectomized female rats and the antioxidant potentials in BV2cells, Arch. Pharm. Res. 5 (2005) 566-572; https://doi.org/10.1007/bf02977760

9. S. S. Joo, T. J. Won, H. C. Kang and D. I. Lee, Isoflavones extracted from Sophorae fructus upregulate IGF-I and TGF-beta and inhibit osteoclastogenesis in rat bone marrow cells, Arch. Pharm. Res. 27 (2004) 99-105; https://doi.org/10.1007/BF02980054

10. D. D. Thompson, H. A. Simmons, C. M. Pirie and H. Z. Ke, FDA guidelines and animal models for osteoporosis, Bone 17 (Suppl. 4) (1995) 125S-133S; https://doi.org/10.1016/8756-3282(95)00285-1

11. K. D. Harrison, B. D. Hiebert, A. Panahifar, J. M. Andronowski, A. M. Ashique, G. A. King, T. Arnason, K. J. Swekla, P. Pivonka and D. M. Cooper, Cortical bone porosity in rabbit models of osteoporosis, J. Bone Min. Res. 35 (2020) 2211-2228; https://doi.org/10.1002/jbmr.4124

12. L. Krenn and V. Pötsch, An efficient HPLC method for the quantification of isoflavones in soy extracts and soy dietary supplements in routine quality control, Pharmazie 61 (2006) 582-585.

13. N. R. Wanderman, C. Mallet, H. Giambini, N. Bao, C. Zhao, K. N. An, B. A. Freedman and A. Nassr, An ovariectomy-induced rabbit osteoporotic model: A new perspective, Asian Spine J. 12 (2018) 12-17; https://doi.org/10.4184/asj.2018.12.1.12

14. F. Öztürk, H. Babacan and C. Gümüş, Effects of zoledronic acid on sutural bone formation: a computed tomography study, Eur. J. Orthodont. 34 (2012) 141-146; https://doi.org/10.1093/ejo/cjq160

15. E. S. Manas, Z. B. Xu, R. J. Unwalla and W. S. Somers, Understanding the selectivity of genistein for human estrogen receptor-beta using X-ray crystallography and computational methods, Structure 12 (2004) 2197-2207; https://doi.org/10.1016/j.str.2004.09.015

16. O. Korb, T. Stützle and T. E. Exner, Empirical scoring functions for advanced protein-ligand docking with PLANTS, J. Chem. Inf. Model. 49 (2009) 84-96; https://doi.org/10.1021/ci800298z

17. G. G. Kuiper, J. G. Lemmen, B. Carlsson, J. C. Corton, S. H. Safe, P. T. van der Saag, B. van der Burg and J. A. Gustafsson, Interaction of estrogenic chemicals and phytoestrogens with estrogen receptor beta, Endocrinology 139 (1998) 4252-4263; https://doi.org/10.1210/endo.139.10.6216

18. P. Thangavel, A. Puga-Olguín, J. F. Rodríguez-Landa and R. C. Zepeda, Genistein as potential therapeutic candidate for menopausal symptoms and other related diseases, Molecules 24 (2019) Article ID 3892; https://doi.org/10.3390\%2Fmolecules24213892

19. F. Lizcano and G. Guzmán, Estrogen deficiency and the origin of obesity during menopause, Biomed. Res. Int. 2014 (2014) Article ID 757461; https://doi.org/10.1155/2014/757461

20. M. Akhlaghi, M. Zare and F. Nouripour, Effect of soy and soy isoflavones on obesity-related anthropometric measures: A systematic review and meta-analysis of randomized controlled clinical trials, Adv. Nutr. 8 (2017) 705-717; https://doi.org/10.3945/an.117.015370

21. Mayo Clinic Laboratories, Clinical Information on Serum Osteocalcin, Test ID: OSCAL; https://www. mayocliniclabs.com/test-catalog/Clinical+and+Interpretive/80579\#: :text=Osteocalcin\%20levels $\% 20$ are $\% 20$ increased $\% 20$ in,fractures $\% 2$ C $\%$ acromegaly $\% 20$ and $\% 20$ bone $\% 20$ metastasis; last access date November 12, 2020.

22. C.-T. Che, M. S. Wong and C. W. Lam, Natural products from Chinese medicines with potential benefits to bone health, Molecules 21 (2016) Article ID 239; https://doi.org/10.3390\%2Fmolecules21030239

23. H. J. Yoon, C. R. Seo, M. Kim, Y. J. Kim, N. J. Song, W. S. Jang, B. J. Kim, J. Lee, J. W. Hong, C. W. Nho and K. W. Park, Dichloromethane extracts of Sophora japonica L. stimulate osteoblast differentiation in mesenchymal stem cells, Nutr. Res. 33 (2013) 1053-1062; https://doi.org/10.1016/j. nutres.2013.08.004

24. C. Picherit, V. Coxam, C. Bennetau-Pelissero, S. Kati-Coulibaly, M. J. Davicco, P. Lebecque and J. P. Barlet, Daidzein is more efficient than genistein in preventing ovariectomy-induced bone loss in rats, J. Nutr. 130 (2000) 1675-1681; https://doi.org/10.1093/jn/130.7.1675 